\title{
The Summary of the Cache Management Strategy about the Distributed VOD System Based on P2P Architecture
}

\author{
Xie Wen-hao \\ Xi'an Shiyou University \\ Department of Applied Mathematics \\ Xi'an, China \\ 1609632928@qq.com
}

\begin{abstract}
On the basis of analyzing the distributed VOD system architecture, this paper introduces the cache management strategies and cache replacement algorithm. And it explains the cache replacement algorithm of the distributed VOD system based on P2PVOD framework. On this basis, it further discusses various cache optimization strategies of the distributed VOD system in recent years. In addition, it has analyzed the characteristics of the existing policies. At last, the paper introduces their application and development prospects.
\end{abstract}

Keywords-P2P; VOD; CDN;distributed VOD system; cache management strategy; cache replacement algorithm.

\section{INTRODUCTION}

P2P network is such the network that P2P streaming media technology is introduced into the field of network streaming media. It makes full use of the calculation, bandwidth and storage resources of the common host nodes, in which nodes obtain the streaming media data from the other nodes, at the same time, also send data to other nodes. P2P streaming media technology has the advantages of IP multicast and easy implementation and deployment without changing the existing Internet structure. It combines P2P network and VOD system, greatly increases the sustaining users number of video on demand system. At the same time, it can improve the quality of the video showing broadcast on the client. It also has good scalability, high bandwidth utilization, the advantages of the low server bandwidth costs so that it has been widely largely used by Internet streaming media service providers.

Based on the analysis and research of the current P2P streaming media on demand system cache management strategy and the distributed VOD system based on P2P architecture cache management mechanism, this paper introduces the distributed VOD system cache management optimization strategy in recent years. At last, it has analyzed the application prospects.

\section{THE SUMMARIZE OF THE DISTRIBUTED VOD SYSTEM}

\section{A. The distributed VOD system}

VoD (video-on-demand) system is an interactive video on demand system, and has a fast forward, fast rewind, pause, and random access video operating characteristics. The randomness and uncertainty of the network users access, as well as the characteristics of large quantities of data require that the streaming media server has high stability and large handing capacity. These features in the video on demand (VOD) system are especially prominent [1].

CDN is namely the Content Delivery Network. The basic idea is as much as possible to avoid the bottlenecks. It can also prevent the nodes to influence the stability of data transmission speed so that it can make content transmission faster, more stable. As the same time, it avoids the disadvantage of the small handing capacity and high latency of the traditional $\mathrm{C} / \mathrm{S}$ architecture caused by the single node performance bottlenecks.

CDN system can timely response the requests of the users to the closest service nodes according to the integrated information such as network traffic, the connections between the nodes, loading condition and the distance to the users. The purpose is to make the users obtain the content to the nearest, solve the status of the Internet network congestion, improve the response speed of the users to access the website.[2,3] On the other hand, if every proxy server caches all of the data, it can bring huge storage costs to the $\mathrm{VoD}$ system because of the large video file data. So it can better solve the problem by using part of the cache and nodes collaboration method. Therefore, CDN and P2P (peer-topeer) structure can make full use of the advantages of two overlay network and reduce the service delay, network jitter and cache space[4].

\section{B. A distributed VOD system architecture}

As shown in figure1, the P2PVoD system studied in this paper is mainly composed of the following parts: the source server and the directory server (tracker server), and peer nodes. The source server saves the entire film. The film is divided into multiple segments in order to the storage and transmission according to the fixed time or the size. Directory services (tracker server) are responsible for maintaining all the nodes in the system of information, including time, broadcast schedule and so on. When a node enter into the system, the node active directory server connects and sends your information (including which film to watch; from which pieces played; IP address and port number, etc.) Directory server returns a list of nodes according to the information. In this system, each node maintains a cache bitmap which recorded the fragmentation 
of the current playing video information and cached fragments. On the basis of the information, the nodes choose the network from the node list returned by the directory server.

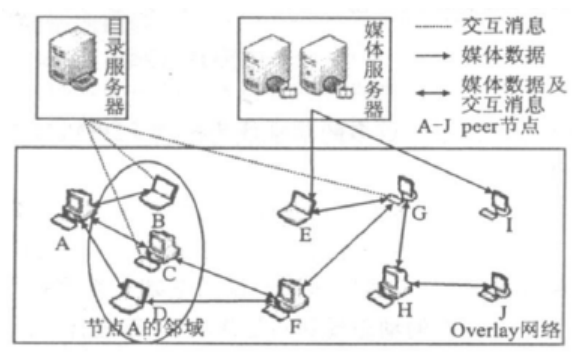

Fig.1. Basic architecture of P2P system

\section{The distributed VOD system cache replacement algorithm}

The cache replacement algorithm is a main work of streaming media caching system, its good or bad directly determines the performance of streaming media caching system. All cache replacement algorithms all cache the new data objects into memory until the cache is full. After that, if it will cache new data objects, it needs to remove some old data objects. The distinction of different algorithms is how to select the recycling target. Now more mature algorithms [5] are following:

- SCU algorithm: it is aimed at improving the cache prefix byte hit ratio in order to reducing access latency and cutting down the streaming media network transmission cost.

- The segmentation strategy of exponential growth: it can quickly replace the segments to fit for the changes in the cache object access patterns.

- LFU and LRU algorithms: they are the models that are respectively considering the sight feature and access frequency. However, there are cache pollution problems for LFU and long ring mode problems for LRU. At the same time, the two kinds of algorithms are also likely to continue to replace the same media objects which resulted in increasing the probability of file cache content to be released in full and response latency and reduced the request hit ratio.

From different extent, although these algorithms improved the cache space utilization and byte hit ratio, they only take account of the single factor. So now there is not such algorithm which is not only suitable for constant bit rate video but also applies to the efficient variable bit rate video. Therefore considering comprehensive factors, to achieve a common efficient cache management strategy is very necessary.

\section{THE DISTRIBUTED VOD SYSTEM CACHE OPTIMIZATION STRATEGY}

\section{A. Layered cache management strategy}

The management of the storage resources distributed VoD system mainly studies how to carry out data push and replacement in order to achieve:

1) Availability of data security;

2) The load balancing of the nodes;

3) Efficient resource location;

4) Video of the granular random access and low latency.

Relative to full-text caching, fragment caching will split large media file into several data segments in order to flexible management. The caching problem in literature [6] for heterogeneous network puts forward a method based on weight. In streaming media system data segments described as heat [7], the weights of it is as a basic parameter to reflect the statistical regularity of users on demand behavior. The randomness of the users access behavior determines the heat complex change [8]. Heat estimates is due to user behavior, it directly affects the effect of caching algorithm. The calculation of heat current usually adopts the way of fixed cycle which cannot reflect the actual changes in real time. Literature [9] by defining the time window and the number of on-demand window statistical data request rate and heat, it can track users behavior change.

\section{B. A cache optimization strategy based on node's life}

In recent years, there have been many scholars to do some research work for availability cache optimization. Represented in the literature [10] assumes that the nodes of the job at a fixed or failure probability to leave the system, and then on the basis of the hypothesis of cache optimization will be described as a standard dynamic programming problem and solution.

Firstly, assume that the node with a fixed probability leaving the system does not meet the P2PVoD application user behavioral characteristics: Based on P2P applications, although a single node leaving system is random, but its overall dynamic behavior is presented a kind of statistical properties of heavy-tailed feature [11].

Secondly, the proposed strategy requires accurate statistics on all kinds of information in the system, and the need to coordinate all the behavior of the user node and its scalability is not strong. Literature [12] puts forward a kind of lazy replication strategy framework to optimize fragments of availability, Probability of each node by leaving the predictor and fragment popularity predictor decide when to copy what fragment information such as the number of copies, replication, and the target node. However, its lifetime prediction method which is put forward using threshold decision mechanism makes the prediction error large.It has not evaluated reasonably the fragments of availability and service efficiency.

Literature [13] proposed a probability cache strategy, according to the fragment as a priority for distances from the playing position of cache optimization. But the mechanism doesn't consider the relationship of the fragment popularity 
and cache availability. It lacks the consideration for caching service efficiency.

In a word, optimizing the system cache availability needs to consider two key questions:(1) evaluating effectively the availability of the fragment and service efficiency; (2) having good extensibility and ease of deployment.

For the above two key problems, the literature [14] considers the availability and service efficiency which is on the basis of analyzing the concept of relative availability. It puts forward a practical method to measure the relative availability which is based on the observation of the nodes lifetime distribution.

\section{The design and implementation of Multistage P2P architecture} [15]

There are three models of P2P technology in architecture:

1) Centralized directory structure: its advantages are simple maintenance and high efficiency,but its disadvantage is that the reliability and security is low, not suitable for large-scale network applications.

2) Pure P2P network structure: Its advantages is good fault tolerance.Its disadvantage is that largely consuming the bandwidth,easily causing network congestion and even instability.

3) Hybrid network structure: The advantage of the structure is better performance and scalability, easier to be managed. Its defect is the strong dependence of the nodes so that its fault tolerance is susceptible.

In order to overcome the above shortcomings existing in the three kinds structure, literature [16] proposes a VOD system based on multistage P2P architecture. It has designed and implemented the distributed node in the system so that the system has good performance. The multistage P2P architecture has five kinds of $\mathrm{P} 2 \mathrm{P}$ nodes which constitute the double P2P network.

\section{THE ANALYSIS OF APPLICATION PROSPECTS}

The randomness of the real-time streaming media service and user behavior bring challenge to the development of large-scale VoD system. This paper introduces the structure of the distributed VOD system and the cache replacement algorithm based on the CDN-P2P architecture, and it further explains the distributed VOD system cache optimization strategy in recent years.

The rapid development and popularization of the Internet has provided the development of P2P streaming media business with the powerful market power, and the streaming media business is becoming increasingly popular. In recent years, the application of distributed VOD system has obtained rapid development and P2P streaming media technology is increasingly mature.

\section{ACKNOWLEDGMENT}

This research was financially supported by the Shaanxi Province Education Department Foundation (Grant NO.15JK1587) and the Youth Innovation Foundation for Xi’an Shiyou University (Grant NO.115030108).

\section{REFERENCES}

[1] Marwan Krunz and Satish K. Tripathi, Impact of video scheduling on bandwidth allocation for multiplexed MPEG streams, Multimedia Systems, Volume 5, Number 6, Nov 2005:80-92.

[2] Shim J, Scheuermann P, Vingralek R. Proxy cache algorithms: design , implementation, andperformance [ J ] . IEEE Trans. Knowledge and Data Engineering, 1999, 11(4) : 549-562.

[3] Liu Jiang-chuan, Xu Jian-liang. Proxy caching for media streaming over the internet $[\mathrm{J}]$. IEEE Communications Magazine, 2004, 42( 8) :88-94.

[4] Borst S, Gupta V, Walid A. Self-organizing algorithms for cache cooperation in content distribution networks $[\mathrm{J}]$. Bell Labs Technical Journal, 2009, 14( 3) :113-126.

[5] Xie Wen-hao. The Summarize of Cache Management Strategy and Cache Replacement Algorithm In P2P Streaming Media Requesting Program System[C].Proceeding of 2015 International Conference on Information,Business and Management,2015:700.

[6] Hsu T H, Li Y H. A weighted segment-based caching algorithm for video streaming objects over heterogeneous networking environments $[\mathrm{J}]$. Expert Systems with Applications, 2011, 38 ( 4 ) :3467-3476.

[7] Li Peng, Zheng Wei, Zhang Kai-hui. The design of streaming media proxy server based on patching first algorithm [C] . Proceedings of the 6th International Forum on Strategic Technology, Harbin: IEEE Harbin Section, 2011: 643-647.

[8] Luo J G, Zhang Q, Tang Y, et al. A trace-driven approach to evaluate the scalability of P2P-based video-on-demand service [J]. IEEE Trans. Parallel and Distributed Systems, 2009, 20(1) : 59-70.

[9] Wu Ya-min,Yin Bao-qun,Zhang Guo-rong,Hu Han. A kind of hierarchical distributed VoD system cache management strategy [J] Journal of Chinese Computer Systems,2013,7(34):1548-1551.

[10] Guo Lei,Chen Song-qing,ZhangXiao-dong.Design and evaluation of as calable and reliable P2P assisted proxy for on-demand streaming media delivery[J] . IEEE Transactionon Knowledge and DataEngineering, May2006, 18(5):64-70.

[11] YeTian, DiWu, Kam-WingNg.An ovelcaching mechanism for peerto-peer based media-on-demand streaming[ J] .Journal of Systems Architecture,2008, 54:55-69.

[12] Chen Jun, Du Xu,Zhuang Xin-jie. A kind of optimization cache strategy based on nodes' life of P2PVoD system[J], Journal of Chinese Computer Systems, 2010,8(31):101-105.

[13] Zhao Peng, YangZong-kai, ChenJing-wen,etal. Design and evaluation of ascalable P2P video on demand system[J] .Journal of Chinese Computer Systems, 2009, 130(9):1730-1733.

[14] Peng Kai, Wu Juan, Yang Zong-kai, etal.Research and prospect On P2P live streaming system[ J] .ComputerScience, 2009, 36(1):10-15.

[15] WangXiao-ming, YaoZhong-mei, DmitriL.Residual-based estimation of peer and link lifetime sin P2P networks[J] .IEEE/ACM Transactionson Networking, 2009, 17(3):726-739.

[16] Wang Song,Ling Qing,Wu gang,etal.The design and implementation of multistage P2P architecture of VOD distrituted nodes system. Journal of Chinese Computer Systems,2011,4(32):27-35. 